\title{
OAM-enhanced transmission for multimode short-range links
}

\section{Tatarczak, Anna; Usuga Castaneda, Mario A.; Tafur Monroy, Idelfonso}

\section{Published in:}

Fiber Lasers XI: Technology, Systems, and Applications. Proceedings

Link to article, DOI:

$10.1117 / 12.2079795$

Publication date:

2015

Document Version

Publisher's PDF, also known as Version of record

Link back to DTU Orbit

Citation $(A P A)$ :

Tatarczak, A., Usuga Castaneda, M. A., \& Tafur Monroy, I. (2015). OAM-enhanced transmission for multimode short-range links. In A. K. Srivastava (Ed.), Fiber Lasers XI: Technology, Systems, and Applications.

Proceedings (Vol. 9390). [93900E] SPIE - International Society for Optical Engineering. Proceedings of SPIE The International Society for Optical Engineering https://doi.org/10.1117/12.2079795

\section{General rights}

Copyright and moral rights for the publications made accessible in the public portal are retained by the authors and/or other copyright owners and it is a condition of accessing publications that users recognise and abide by the legal requirements associated with these rights.

- Users may download and print one copy of any publication from the public portal for the purpose of private study or research.

- You may not further distribute the material or use it for any profit-making activity or commercial gain

- You may freely distribute the URL identifying the publication in the public portal 


\title{
OAM-enhanced transmission for multimode short-range links
}

\author{
Anna Tatarczak, Mario A. Usuga, and Idelfonso Tafur Monroy \\ DTU Fotonik, Technical University of Denmark, 2800 Kgs. Lyngby, Denmark
}

\begin{abstract}
We propose, experimentally demonstrate, and evaluate the performance of a multimode (MM) transmission fiber data link which is based on orbital angular momentum (OAM) modes. The proposed scheme uses OAM modes to increase capacity or reach without recurring to mode division multiplexing (MDM) or special fibers: we first excite an OAM mode and couple it to a $50 \mathrm{~m}, 100 \mathrm{~m}, 200 \mathrm{~m}$ and $400 \mathrm{~m}$ MM fibers. We compare three OAM modes and a conventional optical multimode under the same launch and received optical power conditions. The proposed OAM based solution is a promising candidate for the data centers interconnects and short range links that employ the existing multimode fiber infrastructure.
\end{abstract}

Keywords: Optical Communication, Data Center Interconnects, OAM

\section{INTRODUCTION}

Vertical cavity surface emitting lasers (VCSELs) are the dominant optical sources in the data center's interconnects due to their low power consumption and a small footprint. In particular, $850 \mathrm{~nm}$ VCSELs together with OM3 multi mode fiber (MMF) or OM4 MMF are the base of the short-range links, widely employed in the existing data center infrastructures. The main restraint in this type of links is the intermodal dispersion, which limits the transmission distance to a few hundred meters for bitrates of $10 \mathrm{Gbps}$. IEEE 802.3ae 10G Ethernet standard specifies $300 \mathrm{~m}$ as the multimode span length for $10 \mathrm{Gbps}$ transmission and IEEE 802.3ba 40G/100G Ethernet gives $75 \mathrm{~m}$ as the maximum transmission length required at $40 \mathrm{Gbps}$. Due to the increasing lengths of connections between buildings in data centers there is a need for longer reach multimode interconnects supporting high bit-rates. ${ }^{1}$ Several ways of achieving further distances with $850 \mathrm{~nm}$ VCSELs have been presented in the literature. First approach involves mode filtering of a multi-mode VCSEL. This results in a VCSEL that emits single or quasi-single fundamental transverse mode with a high side-mode suppression ratio of e.g. $16 \mathrm{~dB},{ }^{2}$ $22 \mathrm{~dB},{ }^{3}$ or $30 \mathrm{~dB} .{ }^{4}$ The mode-filtering is performed within the VCSEL structure. Error-free transmission with the single mode $850 \mathrm{~nm}$ VCSEL is achieved over $1 \mathrm{~km}$ OM4 MMF at $25 \mathrm{Gbps},{ }^{5}$ over $1.1 \mathrm{~km}$ OM4 MMF at $20 \mathrm{Gbps},{ }^{6}$ over $600 \mathrm{~m} \mathrm{OM} 3+\mathrm{MMF}$ at $25 \mathrm{Gbps}^{7}$ and over $50 \mathrm{~m} \mathrm{OM} 3+\mathrm{MMF}$ at $40 \mathrm{Gbps}$. A second approach is to couple the light from a multimode VCSEL to a standard single mode fiber (SSMF). Adding a mode filter to remove $L P_{11}$ mode allows transmission distance of $1 \mathrm{~km}$ SSMF at $10 \mathrm{Gbps}{ }^{8}$ The third approach involves using special $850 \mathrm{~nm}$ optimized singlemode fiber, e.g. photonic crystal fiber $(\mathrm{PCF})$. The core region of $\mathrm{PCF}$ is surrounded by multiple air holes, thus assuring single mode operation. Transmission over $3 \mathrm{~km} \mathrm{PCF} \mathrm{has} \mathrm{been}$ presented at $10 \mathrm{Gbps}^{9}{ }^{9}$ For the multimode VCSELs over multimode fiber, error free transmission over $200 \mathrm{~m}$ has been achieved at 25 Gbps. ${ }^{10}$ All these approaches require substantial modifications of the existing data center infrastructure: replacement of the multimode sources in the first approach, or the fibers in the second and third approaches. Therefore, an alternative solution that employs the existing fibers and VCSELs is of interest.
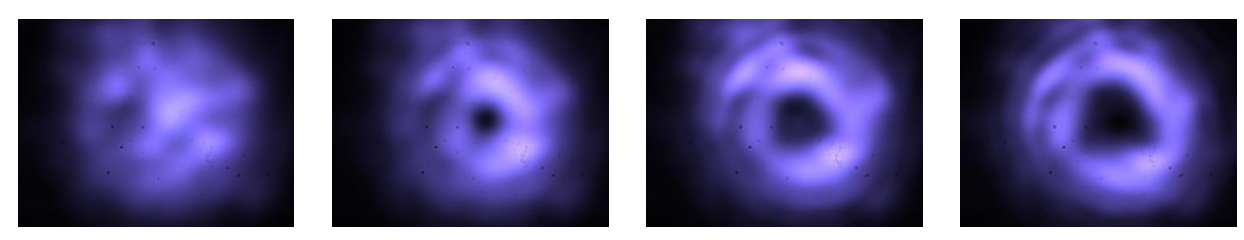

Figure 1. Orbital angular momentum (OAM) modes captured with the camera after spatial light modulator (SLM): M0 (left), M1, M2 and M3 (right); M0 is a standard optical mode.

Next-Generation Optical Networks for Data Centers and Short-Reach Links II, edited by Atul K. Srivastava, Proc. of SPIE Vol. 9390, 93900E · C 2015 SPIE · CCC code: 0277-786X/15/\$18 · doi: 10.1117/12.2079795 


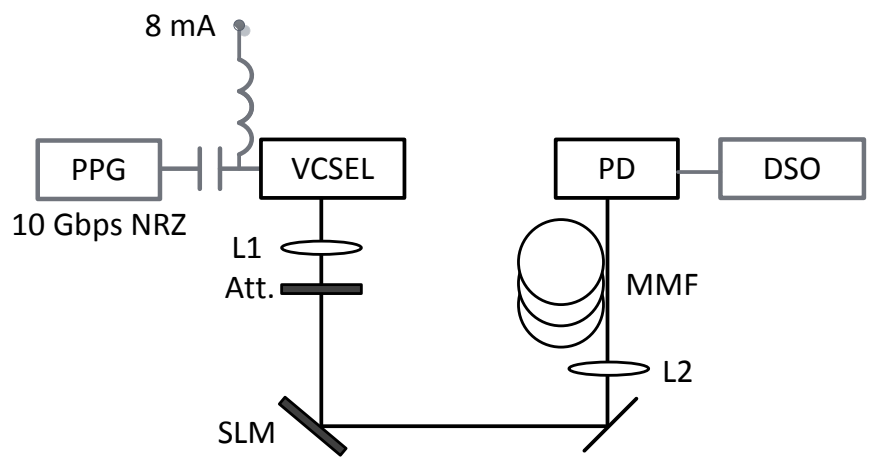

Figure 2. Experimental setup for the transmission of the orbital angular momentum (OAM) modes over multi mode fiber (MMF); Signal from pulse pattern generator (PPG) directly modulates vertical cavity surface-emitting laser (VCSEL) biased at $8 \mathrm{~mA}$; the VCSEL beam is collimated at lens L1 and in free-space passes through the attenuator to the spatial light modulator (SLM), where the beam is shaped to the OAM mode; the shaped beam is coupled to the $3 \mathrm{~m}$ OM3 MMF patchcord; Lens L2 is used for the coupling; The signal is then transmitted through the multi mode fiber (MMF): $3 \mathrm{~m}$ back to back (B2B), $50 \mathrm{~m}$ OM3, $50 \mathrm{~m}$ OM4, $100 \mathrm{~m}$ OM3, $200 \mathrm{~m}$ OM3, $400 \mathrm{~m}$ OM3, $400 \mathrm{~m}$ OM4 and $400 \mathrm{~m}$ customized OM4 by Draka; the signal is then received with photodiode (PD) with the inbuilt transimpedance amplifier (TIA) and the data is stored at digital storage oscilloscope (DSO).

In this paper we propose an approach to increase the obtainable transmission distance for the multi-mode sources over multi-mode fibers. Shaping a multimode VCSEL beam as an orbital angular momentum (OAM) mode (Fig. 1) enabled achieving performance below forward error correction (FEC) threshold during transmissions at $10 \mathrm{Gbps}$ over $400 \mathrm{~m}$ MMF. We provide a comparison between transmission of OAM modes M1, M2, and M3 and standard optical mode M0. The modes are transmitted over: 50 m OM3, $50 \mathrm{~m}$ OM4, $100 \mathrm{~m}$ OM3, $200 \mathrm{~m}$ OM3, $400 \mathrm{~m}$ OM3, $400 \mathrm{~m}$ OM3, and $400 \mathrm{~m}$ Draka OM4. The last mentioned fiber is a Max-CAP-OM4 fiber designed by Prysmian Group Draka. It will be further referred to as Draka OM4 fiber.

\section{SETUP}

The experimental setup is shown in Fig. 2. The $850 \mathrm{~nm}$ multimode commercially available VCSEL is biased at 8 $\mathrm{mA}$ and directly modulated with $10 \mathrm{Gbps}$ or $11 \mathrm{Gbps}$ pseudo-random bit sequence (PRBS). The sequence length is $2^{15}-1$ and the amplitude is $800 \mathrm{Vpp}$. The LIV curves and optical spectrum of the VCSEL used are presented in Fig. 3 and Fig. 4, respectively. The modulated optical beam from the pigtailed VCSEL is collimated and passed through the variable attenuator to the spatial light modulator (SLM) in the free-space part of the setup. The optical power level is controlled by the variable attenuator. The SLM is used to shape the multimode beam to the OAM mode. We verify the transmission performance with OAM modes M1 to M3 and a conventional multimode M0. For M0, SLM behaves as a mirror. The 4 modes under investigation are captured with the camera after SLM and presented in Fig. 1. The full length of the free-space link is $1.5 \mathrm{~m}$. At the end of the free-space link the beam is coupled into the $1 \mathrm{~m}$ long MMF OM3 patchcord. The coupling after free-space is aligned to obtain maximum output power and is readjusted for each OAM mode.

The OM3 patchcord used for coupling is connected to the multimode fiber spool via the fiber connector. Several MMFs are tested: $50 \mathrm{~m}$ OM3, $50 \mathrm{~m}$ OM4, $100 \mathrm{~m} \mathrm{OM} 3,200 \mathrm{~m}$ OM3, $400 \mathrm{~m} \mathrm{OM} 3,400 \mathrm{~m}$ OM 4 and $400 \mathrm{~m}$ OM4 special fiber by Draka. The loss in $50 \mathrm{~m}, 100 \mathrm{~m}$ and $200 \mathrm{~m}$ is below $0.5 \mathrm{~dB}$. For $400 \mathrm{~m}$ spools the measured loss is: $0.98 \mathrm{~dB}$ for OM3, 0.95 for OM4, and $0.97 \mathrm{~dB}$ for Draka OM4. After transmission the signal is received by the $850 \mathrm{~nm}$ commercially available photodiode (PD) with $25 \mathrm{GHz}$ bandwidth. The signal is then stored at digital storage oscilloscope (DSO) with $14 \mathrm{GHz}$ bandwidth. For each BER point $10^{7}$ symbols are saved and errors are counted. No additional equalization is used.

The optical system frequency response is characterized with the vector network analyzer (VNA) before the transmission is performed. For the $S_{21}$ measurement the output of pulse pattern generator (PPG) is replaced 


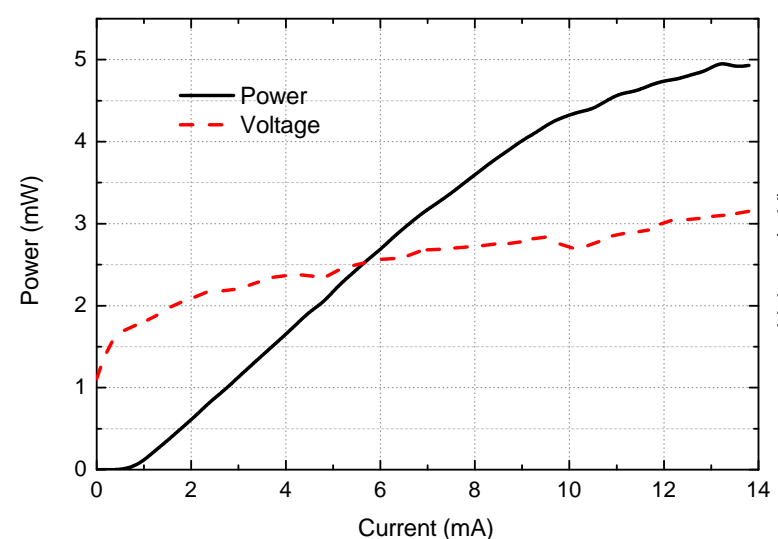

Figure 3. Static characteristics of the VCSEL: Power versus current and voltage versus current (LIV curves).



Figure 4. Optical spectrum of the VCSEL captured after $400 \mathrm{~m}$ of MMF; two major modes are around $858 \mathrm{~nm}$.

with the VNA output and input to DSO is instead received by the VNA input. The optical path, from VCSEL to PD included, is kept the same.

\section{RESULTS}

We compare a transmission performance for different OAM modes (M1, M2, M3) and for a conventional multimode M0. Firstly, the modes M0 - M2 are transmitted at 11 Gbps over $100 \mathrm{~m}$ OM3 and $200 \mathrm{~m}$ OM3. Secondly, the modes M0 - M2 are transmitted over two $50 \mathrm{~m}$ long MMFs, OM3 and OM4, at 11 Gbps. Finally, modes M0 - M3 are transmitted over 3 types of $400 \mathrm{~m}$ MMF. The performance is compared in terms of the bit-error-ratio (BER) relative to the received average power measured before PD. Transmission over $1 \mathrm{~m}$ of OM3 MMF is referred to as back to back (B2B) transmission. Additionally, the $S_{21}$ is measured for each optical system.

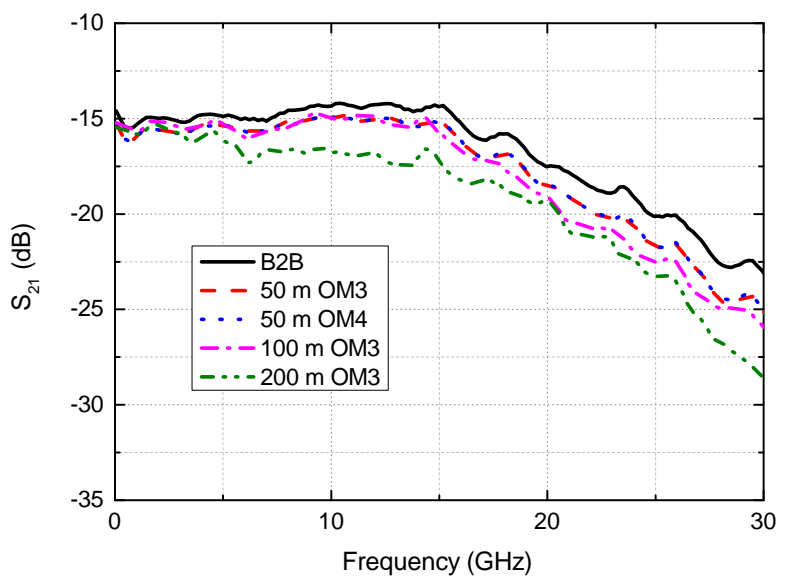

Figure 5. $S_{21}$ measured for the optical system with back to back (B2B) and four different multi mode fiber (MMF): $50 \mathrm{~m} \mathrm{OM} 3,50 \mathrm{~m}$ OM4, $100 \mathrm{~m}$ OM3 and $200 \mathrm{~m} \mathrm{OM} 3$; All curves are measured with a conventional multimode M0.



Figure 6. BER versus received optical power (ROP) measured for back to back (B2B), $100 \mathrm{~m}$ OM3 and $200 \mathrm{~m} \mathrm{OM} 3$ for 2 different orbital angular momentum (OAM) modes: M1 and M2 and a conventional multimode M0. 


\subsection{Transmission over B2B, $100 \mathrm{~m}$ OM3, and $200 \mathrm{~m}$ OM3}

Fig. 5 presents $S_{21}$ measured for the optical system with back to back (B2B), $50 \mathrm{~m}$ MMF, $100 \mathrm{~m}$ and $200 \mathrm{~m}$. All of the $S_{21}$ are measured for a conventional multimode M0. 3-dB bandwidth of the system ranges from $16 \mathrm{GHz}$ for $200 \mathrm{~m}$ to $22 \mathrm{GHz}$ for B2B. The measured analog bandwidths for all presented cases are sufficient for the 11 Gbps transmission. The bit error rate (BER) curves measured at $11 \mathrm{Gbps}$ for $\mathrm{B} 2 \mathrm{~B}$ and all the fibers are presented in Fig. 6. No penalty is observed, neither for different fiber lengths nor for the different modes. The power measurement error due to the procedure is equal to $-/+0.25 \mathrm{~dB}$. Receiver sensitivity at BER of $10^{-3}$ is equal to $-10 \mathrm{dBm}$.

\subsection{OM3 vs. OM4}

In Fig. 7 the comparison between 11 Gbps transmission over $50 \mathrm{~m}$ links, OM3 and OM4 MMF, is presented. As apparent from Fig.5, the bandwidth for both fibers is the same. The measured 3-dB bandwidth is $20 \mathrm{GHz}$ for the length under consideration. The sensitivity at BER $10^{-3}$ for OM4 fiber is up to $0.2 \mathrm{~dB}$ better than for OM3. The same is observed for all of the OAM modes. This difference in the sensitivity is within the power measurement error margin. The difference between OM3 and OM4 is expected to be more significant for longer fiber link.

\section{$3.3400 \mathrm{~m}$}

A link of $400 \mathrm{~m}$ is used to evaluate the transmission performance with the OAM modes. We use three different $400 \mathrm{~m}$ long links in which the intermodal dispersion is dominant. $S_{21}$ curves measured for the optical system with each of the three links are shown in Fig. 8. The $S_{21}$ curves are measured with for M0 OAM mode. The 3-dB bandwidth is only $4.9 \mathrm{GHz}$ for MMF OM4 by Draka, $4.8 \mathrm{GHz}$ for MMF OM3 and $4 \mathrm{GHz}$ for $400 \mathrm{~m}$ MMF OM4.

The impact of the OAM modes on the $S_{21}$ of the system is presented in Fig. 9. The frequency response is measured for OM4 Draka fiber with OAM modes from M1 to M3 and with the conventional multimode M0. The measured responses are the same for modes M0 and M1, while responses of modes M2 and M3 have $\sim 2 \mathrm{~dB}$ less power for frequencies above $15 \mathrm{GHz}$. For each of the modes the coupling is realigned to reach the highest coupled power. The coupling efficiency is a ratio between the power coupled to the MMF OM3 patchcord and the input power to the lens. Fig. 10 presents the coupling efficiency measured for modes M0 to M3. It shows that there is no difference in coupling efficiency for M0 and M1, however for M2 and M3 the coupling efficiency



Figure 7. BER versus received optical power (ROP) measured for 2 OAM modes (M1 and M2) and a conventional multimode M0 for two types of $50 \mathrm{~m} \mathrm{MMF:} \mathrm{OM4} \mathrm{and} \mathrm{OM3.}$

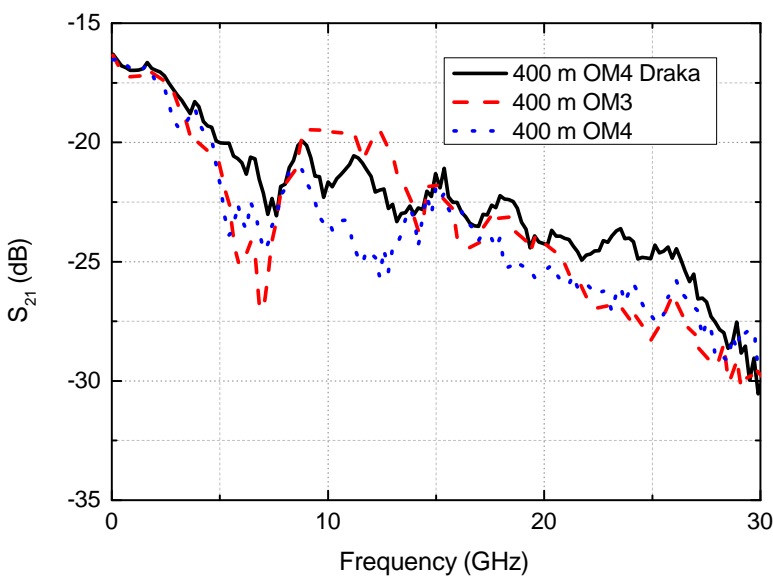

Figure 8. $S_{21}$ measured for the optical system with three $400 \mathrm{~m}$ spools: OM3, OM4 and OM4 fiber by Draka; All curves are measured with a conventional M0 mode. 


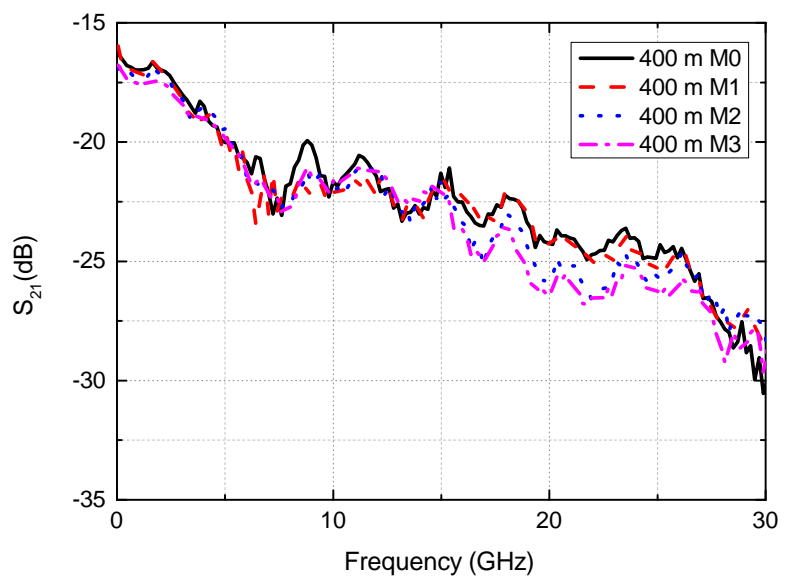

Figure 9. $S_{21}$ measured for the optical system with $400 \mathrm{~m}$ OM4 by Draka with 4 OAM modes: M0, M1, M2 and M3.

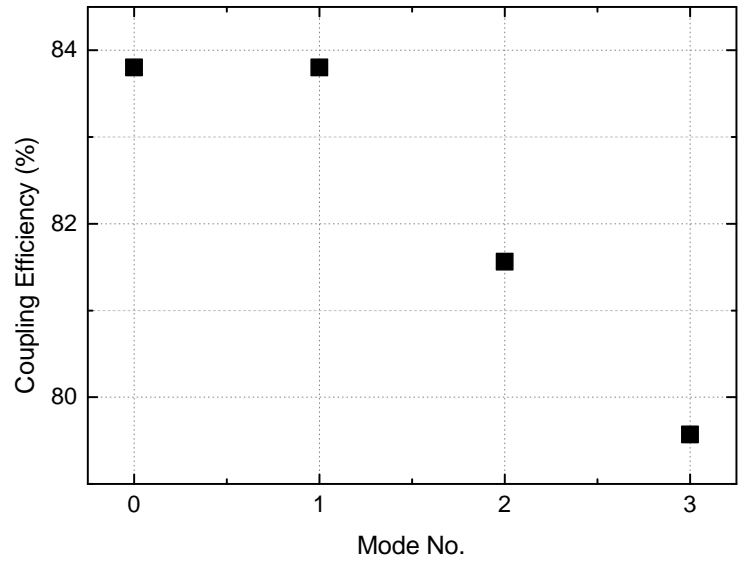

Figure 10. Coupling efficiency measured in the system as $P_{\text {coupled }} / P_{I N}$ for 4 modes.

decreases. Higher order modes suffer from larger loss, as the power is distributed outside of the beam center and the coupling is imperfect.

Fig. 11, Fig. 12 and Fig. 13 depict the BER curves measured at $10 \mathrm{Gbps}$ for three $400 \mathrm{~m}$ links. For all of the curves the same tendency is observed: the higher the order of OAM mode, the lower amount of errors is counted. During the measurement the coupling from the free-space through the second lens L2 is realigned for each mode to reach the highest coupling efficiency. The BER below FEC threshold is obtained by transmitting the beam shaped in OAM mode. The error floor observed at $\log (B E R)$ of -1.8 for $400 \mathrm{~m}$ OM3 link is moved down to -2.1 with M1 and M2 and down to -2.8 with M3. OM4 fiber has the lowest 3-dB bandwidth out of the three links under consideration, as shown in Fig.8. The error floor for this fiber is measured at $\log (B E R)$ of -1.3 . Using OAM mode M1 allows to move the error floor down to $\log (B E R)$ of $-1.5, \mathrm{M} 2$ to -2.3 and M3 to -3.3 . In case of Draka $400 \mathrm{~m}$ OM4 fiber, the 3-dB bandwidth is the highest of the three links (Fig.8). Therefore, the error floor

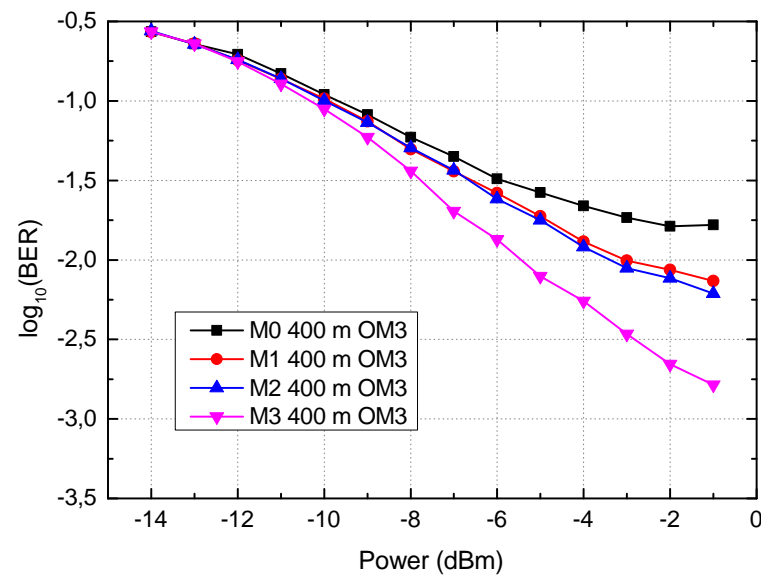

Figure 11. Bit error rate (BER) versus received optical power (ROP) measured at $10 \mathrm{Gbps}$ for $400 \mathrm{~m}$ OM3 multi mode fiber (MMF) for 3 OAM modes (M1, M2, and M3) and a conventional multi mode M0.



Figure 12. Bit error rate (BER) versus received optical power (ROP) measured at $10 \mathrm{Gbps}$ for $400 \mathrm{~m}$ OM4 multi mode fiber (MMF) for 3 OAM modes (M1, M2, and M3) and a conventional multi mode M0. 


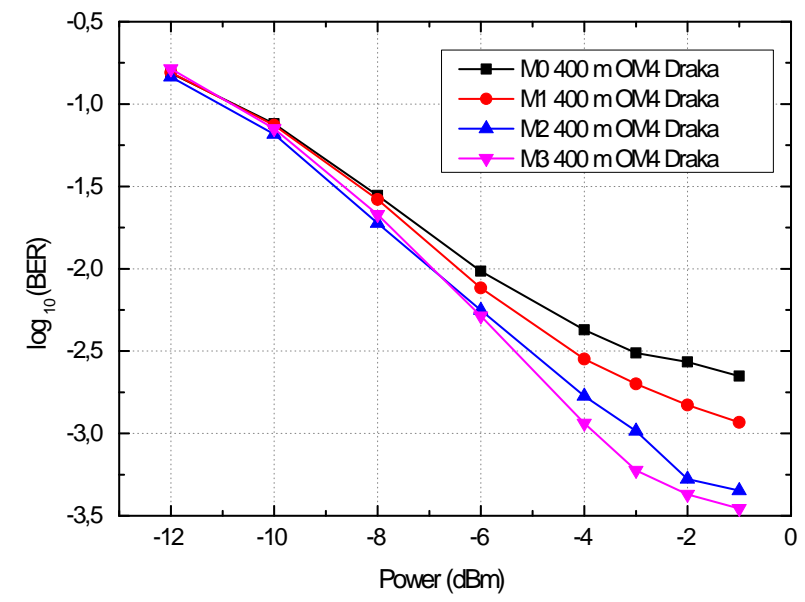

Figure 13. Bit error rate (BER) versus received optical power (ROP) measured at 10 Gbps for $400 \mathrm{~m}$ OM4 multi mode fiber (MMF) by Draka for 3 OAM modes (M1, M2, and M3) and a conventional multi mode M0.

for M0 is the lowest, at $\log (B E R)$ of -2.6 . Usage of M3 allows reaching -3.45 .

\section{DISCUSSION}

As shown in Fig. 6, using the OAM modes M1 and M2 over $100 \mathrm{~m}$ OM3 and $200 \mathrm{~m}$ OM3 results in the same performance in terms of BER as using a conventional multimode M0. The same transmission performance within the tested BER range indicates a potential for simultaneous use of several OAM modes in the future spatial division multiplexing (SDM) systems.

For $400 \mathrm{~m}$ transmission, 3-dB system bandwidth becomes a limiting factor, as presented in Fig. 8 . The 'dip' in the frequency response occurs due to the interaction between several modes in the fiber. It is the most pronounced for the OM3 fiber and the least for the Draka's OM4 fiber. The 3-dB bandwidth of 4 GHz measured for $400 \mathrm{~m}$ OM4 fiber results in the error floor measured at $\log (B E R)$ of -1.3 for $10 \mathrm{Gbps}$ transmission. Using OAM modes introduces the most significant performance improvement for $400 \mathrm{~m}$ OM4 fiber. Using OAM mode M3 allows to reach error floor below FEC threshold, at $\log (B E R)$ of -3.3. For Draka OM4 fiber, the measured 3 -dB bandwidth was $4.9 \mathrm{GHz}$. It allows for the error floor below $\log (B E R)$ of -2.6. Using OAM mode M3 enables $\log (B E R)$ of -3.45 . An improvement in the transmission performance while using higher order OAM modes is measured for all $400 \mathrm{~m}$ fiber types with the best performance of OAM mode M3 transmitted over Draka OM4 MMF.

\section{FUTURE WORK}

The presented results provide an interesting solution for improving performance in terms of BER in the multimode transmission scenarios. A further study is required to define the optimal launching and transmission conditions. The next step is to create an all fiber coupled OAM-enhanced transmission, with no free-space link. Secondly, higher order modes M4 - M6 are expected to result in further performance improvement, hence need to be tested. Additionally, checking the behavior of the OAM enhanced transmission with a single mode source should be verified and compared with the presented results.

\section{CONCLUSION}

In this work we present a novel OAM-enhanced transmission over multi mode fiber (MMF) which can be applied in the short-range optical interconnects. OAM modes M1 and M2 perform the same as conventional multi mode 
M0 within the tested BER range for transmission over $50 \mathrm{~m} \mathrm{OM} 3,50 \mathrm{~m}$ OM4, $100 \mathrm{~m} \mathrm{OM} 3,200 \mathrm{~m}$ OM3. For a further distance of $400 \mathrm{~m}$, the higher order modes perform better than the conventional M0 mode, enabling BER below FEC threshold at $10 \mathrm{Gbps}$ in the system with 3-dB bandwidth of $4 \mathrm{GHz}$.

\section{REFERENCES}

1. C. Lam, H. Liu, B. Koley, X. Zhao, V. Kamalov, and V. Gill, "Fiber optic communication technologies: What's needed for datacenter network operations," Communications Magazine, IEEE 48, pp. 32-39, July 2010.

2. P. Moser, J. Lott, P. Wolf, G. Larisch, H. Li, N. Ledentsov, and D. Bimberg, "56 fJ dissipated energy per bit of oxide-confined $850 \mathrm{~nm}$ VCSELs operating at 25 Gbit/s," Electronics Letters 48, pp. 1292-1294, September 2012.

3. P. Wolf, P. Moser, G. Larisch, H. Li, J. Lott, and D. Bimberg, "119 fJ of Dissipated energy per bit for errorfree $40 \mathrm{Gbit} / \mathrm{s}$ transmission across $50 \mathrm{~m}$ of multimode optical fiber using energy efficient $850 \mathrm{~nm}$ VCSELs," in Lasers and Electro-Optics (CLEO), 2013 Conference on, pp. 1-2, June 2013.

4. F. Mederer, C. Jung, R. Jager, M. Kicherer, R. Michalzik, P. Schnitzer, D. Wiedenmann, and K. Ebeling, "12.5 Gbit/s data rate fiber transmission using single-mode selectively oxidized GaAs VCSELs at $\lambda=850$ nm," in LEOS '99. IEEE Lasers and Electro-Optics Society 1999 12th Annual Meeting, 2, pp. 697-698 vol.2, 1999.

5. M. P. Tan, S. Fryslie, J. Lott, N. Ledentsov, D. Bimberg, and K. Choquette, "Error-Free Transmission Over 1-km OM4 Multimode Fiber at $25 \mathrm{~Gb} / \mathrm{s}$ Using a Single Mode Photonic Crystal Vertical-Cavity SurfaceEmitting Laser," Photonics Technology Letters, IEEE 25, pp. 1823-1825, Sept 2013.

6. R. Safaisini, K. Szczerba, E. Haglund, P. Westbergh, J. Gustavsson, A. Larsson, and P. Andrekson, "20 Gbit/s error-free operation of $850 \mathrm{~nm}$ oxide-confined VCSELs beyond $1 \mathrm{~km}$ of multimode fibre," Electronics Letters 48, pp. 1225-1227, September 2012.

7. P. Moser, J. Lott, P. Wolf, G. Larisch, A. Payusov, N. Ledentsov, and D. Bimberg, "Energy-Efficient OxideConfined 850-nm VCSELs for Long-Distance Multimode Fiber Optical Interconnects," Selected Topics in Quantum Electronics, IEEE Journal of 19, pp. 7900406-7900406, March 2013.

8. Z. Tian, C. Chen, and D. Plant, "850-nm VCSEL Transmission Over Standard Single-Mode Fiber Using Fiber Mode Filter," Photonics Technology Letters, IEEE 24, pp. 368-370, March 2012.

9. H. Hasegawa, Y. Oikawa, T. Hirooka, M. Yoshida, and M. Nakazawa, "10 Gb/s transmission over $3 \mathrm{~km}$ at $850 \mathrm{~nm}$ using single-mode photonic crystal fiber, single-mode VCSEL, and Si-APD," in Optical Fiber Communication Conference, 2006 and the 2006 National Fiber Optic Engineers Conference. OFC 2006, pp. 3 pp.-, March 2006.

10. J. Kropp, J. Lott, N. Ledentsov, P. Otruba, C. Knochenhauer, and F. Ellinger, " $25 \mathrm{~Gb} / \mathrm{s}$ transmission at 850 $\mathrm{nm}$ on multimode fiber with low cost optical component assemblies," in Semiconductor Conference Dresden (SCD), 2011, pp. 1-4, Sept 2011. 